\title{
Outage Analysis of Cooperative OFDM Systems with Nonlinear Amplifiers and Relay Selection
}

\author{
C. Alexandre R. Fernandes, Daniel Benevides da Costa and André L. F. de Almeida
}

\begin{abstract}
Resumo-Comunicações cooperativas surgiram como uma promissora solução para redes sem fio. Em particular, recentemente tem-se presenciado um interesse crescente em sistemas cooperativos do tipo amplifica-e-encaminha com seleção de relay. Por outro lado, a técnica de multiplexação por divisão em frequências ortogonais (OFDM) tem se mostrado ser uma tecnologia chave para os futuros sistemas de comunicação sem fio. Um dos principais problemas de sistemas OFDM é a grande variação da amplitude dos sinais transmitidos, o que pode levar à introdução de distorções não-lineares devido à presença de amplificadores de potência não-lineares. Neste artigo, uma análise teórica da probabilidade de outage em um sistema OFDM do tipo AF com seleção de relay é desenvolvida, análise esta que leva em conta as distorções não-lineares introduzidas por um amplificador de potência não-linear. A análise mostra como os parâmetros do amplificador alteram a probabilidade de outage para diferentes niveis de SNR. Resultados numéricos são fornecidos para validar os resultados analíticos.
\end{abstract}

Palavras-Chave-Amplifica-e-encaminha, seleção de relay, OFDM, amplificador de potência não-linear, probabilidade de outage.

Abstract-Cooperative communications have emerged as a promising solution for wireless networks. In particular, currently there is a growing interest in amplify-and-forward (AF) cooperative systems with relay selection. On the other hand, orthogonal frequency division multiplexing (OFDM) has shown to be a key technology for future wireless communication systems. One of the main problems of OFDM systems is the high peak-to-average power ratio (PAPR) of the transmitted signals, which may cause the introduction of nonlinear distortions due to the presence of nonlinear power amplifiers (PAs). In this paper, a theoretical analysis of the outage probability of an AF OFDM system with relay selection is developed. Our analysis takes into consideration the nonlinear distortions introduced by a nonlinear PA and shows how its parameters affects the outage probability for different SNR levels. Numerical results are provided to validate the analytical results.

Keywords-Amplify-and-forward, relay selection, OFDM, nonlinear power amplifier, outage probability.

\section{INTRODUCTION}

In the last years, cooperative diversity has emerged as a promising technology for wireless communication systems due to its ability to exploit spatial diversity without the need of multiple antennas. Cooperative diversity systems emulate an antenna array in a distributed manner, allowing one or more

C. A. R. Fernandes and D. B. da Costa are with the Department of Computer Engineering, Federal University of Ceará, Sobral, Brazil. E-mails: alexandrefernandes@ufc.br, danielbcosta@ieee.org. C. A. R. Fernandes is partially supported by FUNCAP/Brazil (Proc. BP1-0031-00106.01.00/10). D. B. da Costa is partially supported by CNPq/Brazil (Proc. 302106/2011-1).

A. L. F. de Almeida is with the Department of Teleinformatics Engineering, Federal University of Ceará, Fortaleza, Brazil. E-mail: andre@gtel.ufc.br. A. L. F. de Almeida is partially supported by CNPq/Brazil (Proc. 303238/2010$0)$. mobile units to relay the information data from a source node to the destination node [1]. Several cooperative relaying protocols have been proposed in the literature, such as amplifyand-forward (AF), fixed decode-and-forward, and selective decode-and-forward [2]. Herein, we are interested in the AF protocol due to the fact that it avoids decoding at the relays and, therefore, it is often preferable when complexity or latency issues are of importance.

Currently, there is a growing interest in AF cooperative systems with relay selection, where multiple relays are available and one or more relays are selected according to some welldefined rule [3]. In this work, we are particularly interested in relay selection based on instantaneous and partial knowledge of the channel, as in [4], where the relay selection depends only on the quality of the source-relays links.

On the other hand, orthogonal frequency division multiplexing (OFDM) has become the basis of many wireless communication standards, such as IEEE 802.11a, IEEE 802.16 and 3GPP LTE [5], mainly due to its high spectral efficiency and low complexity implementation. One of the main problems of OFDM is that the transmitted signals are characterized by a high peak-to-average power ratio (PAPR) [5]. Due to the presence of nonlinear devices such as power amplifiers (PAs), a high PAPR causes the introduction of nonlinear distortions in the received signals, which severely affect receiver performance.

In non-cooperative systems, a high PAPR is usually more critical in uplink transmissions, as user terminals have cheaper equipments and stronger power constraints than base stations. Moreover, base stations have more processing resources than user equipments, allowing for the application of techniques to compensate the amplifier nonlinearity at the base station. Indeed, OFDM-based multiple access scheme has been adopted for the downlink in the 3GPP Long Term Evolution (LTE), while single-carrier frequency division multiple access (SCFDMA) has been adopted for the uplink [5]. In cooperative relay systems, the high PAPR is also an important issue in the downlink, as the nonlinear PA of the relay may introduce significant distortions [6], [7].

In this work, a theoretical analysis of the outage probability of AF cooperative diversity OFDM systems with relay selection is developed by taking the nonlinear distortions introduced by the relay PA into account. We assume a Rayleigh fading and downlink transmission, with the base station having a linear PA and the relay having a nonlinear memoryless PA. Moreover, we consider a maximal-ratio combining (MRC) receiver to combine the signals received through the direct link and through the relay link. Specifically, the cumulative distribution function (CDF) of the output of the MRC is 
derived, assuming that the relay node is selected based only on the source-relay channels.

To the best of authors' knowledge, up to now only few works have investigated the impact of PA nonlinear distortions in cooperative communication systems, but none of them carried out an outage analysis [6]-[8].

The rest of the paper is organized as follows. Section II describes the system model considered in this work. In Section III, the outage analysis is presented. In Section IV, we evaluate the validity of the proposed outage analysis by means of computer simulations. Finally, some conclusions and perspectives are drawn in Section V.

\section{SYSTEM MODEL}

A simplified scheme of the two-hop AF cooperative OFDM downlink used in this work is shown in Fig. 1. Time-division multiple-access (TDMA) is employed for orthogonal channel access, all the nodes are equipped with a single antenna operating in half-duplex mode and the three nodes (i.e, source, selected relay, and destination) are synchronized at the symbol level. A cooperative diversity scenario is assumed, where the source $(\mathrm{S})$ communicates with the destination (D) through a direct link (stage 1) and through the help of one out of $M$ available relays (stage 2), with such selection being based on the instantaneous knowledge of the source-relays channels. With this aim, the source (base station) continuously monitors the quality of the source-relay links using local feedback.

We make some key assumptions regarding the cooperative scenario and channel model:

- As in [4], the relays are clustered relatively close together, which allows us to assume that the source-relay links undergo independent and identically distributed (i.i.d.) fading.

- The cluster of relays is assumed to be close to the source so that the source-relay channels have flat fading. The relay-destination and source-destination channels have frequency-selective fading.

- All the wireless links undergo Rayleigh fading.

- The source's PA is linear while the relays' PAs are nonlinear. This assumption is reasonable in a downlink transmission, as the source is a base station with less power constraints and more processing resources than the relays (user terminals).

Assuming that the length of the OFDM cyclic prefix is greater than or equal to the channel delay spread, the discretetime baseband signal in the frequency-domain $x_{n}^{(S D)}$ received through the direct link (source-destination) at the $n^{\text {th }}$ subcarrier can be written as:

$$
x_{n}^{(S D)}=h_{n}^{(S D)} \sqrt{P_{s}} s_{n}+v_{n}^{(S D)},
$$

for $1 \leq n \leq N$, where $N$ is the number of subcarriers, $h_{n}^{(S D)}$ is the channel frequency response (CFR) at the $n^{t h}$ subcarrier of the source-destination link, $s_{n}$ is the frequency-domain data symbol at the $n^{t h}$ subcarrier and $v_{n}^{(S D)}$ is the corresponding additive white Gaussian noise (AWGN) component in the frequency-domain. The data symbols $s_{n}(1 \leq n \leq N)$ are assumed to be stationary and i.i.d., with an uniform

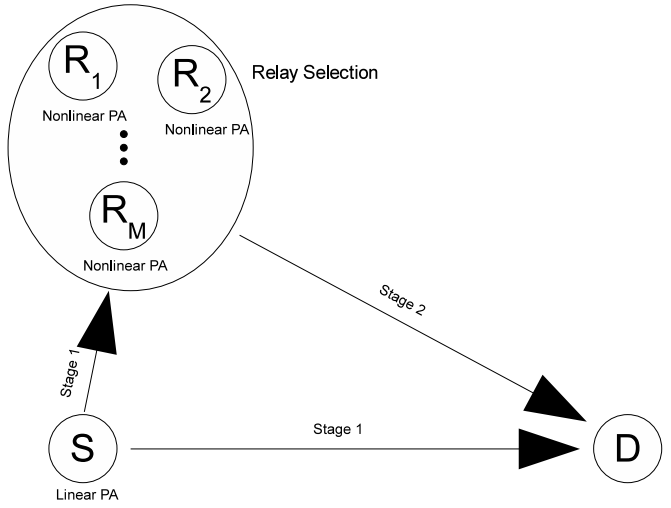

Fig. 1. Cooperative system model.

distribution over a quadrature amplitude modulation (QAM) or phase-shift keying (PSK) alphabet and unitary variance.

Let us denote by $M_{S}$ the index of the selected relay, that is, $M_{S}=\arg _{m} \max \left|h_{m}^{(S R)}\right|^{2} / \sigma_{v}^{2}$, where $1 \leq m \leq M, h_{m}^{(S R)}$ is the complex gain of the flat fading channel linking the source and the $m^{\text {th }}$ relay and $\sigma_{v}^{2}$ is the noise variance, assumed to be the same for all the relays. The signal received by the selected relay at the $n^{t h}$ subcarrier in the frequency-domain is then given by:

$$
x_{n}^{(S R)}=h_{M_{S}}^{(S R)} \sqrt{P_{s}} s_{n}+v_{n}^{(S R)}
$$

where $v_{n}^{(S R)}$ is the corresponding AWGN. At the selected relay, the frequency-domain signal $x_{n}^{(S R)}$ is multiplied by a gain in the following way: $q_{n}^{(R)}=g x_{n}^{(S R)}$, for $1 \leq n \leq N$, where $g$ is the amplification factor. In this paper, we use a variable gain given by [1]: $g=\sqrt{P_{r}} / \sqrt{\left|h_{M_{S}}^{(S R)}\right|^{2} P_{s}+\sigma_{v}^{2}}$, where $P_{r}$ is the transmission power of each relay subcarrier at the input of the PA.

After computing the inverse discrete Fourier transform (IDFT) of $q_{n}^{(R)}(1 \leq n \leq N)$ and inserting the cyclic prefix, the time domain version of $q_{n}^{(R)}(1 \leq n \leq N)$ is amplified by a PA that is modeled as memoryless function $F(\cdot)$. Thus, it follows that:

$$
\tilde{z}_{n^{\prime}}^{(R)}=F\left(\tilde{q}_{n^{\prime}}^{(R)}\right)
$$

for $1 \leq n^{\prime} \leq N+M_{C P}$, where $\tilde{q}_{n^{\prime}}^{(R)}$ and $\tilde{z}_{n^{\prime}}^{(R)}$ are the timedomain signals at the input and output of the PA, respectively, and $M_{C P}$ is the cyclic prefix length.

For a high number $N$ of subcarriers, the signal transmitted by the source in the time-domain $\tilde{s}_{n^{\prime}}$ can be modeled as a complex Gaussian random variable. Therefore, $\tilde{q}_{n^{\prime}}^{(R)}$ is also a complex Gaussian random variable. Thus, assuming a rectangular pulse shaping at the transmission and using the extension of Bussgang's theorem to bandpass memoryless nonlinearities with complex Gaussian inputs, the PA output in the timedomain can be expressed as [9]:

$$
\tilde{z}_{n^{\prime}}^{(R)}=K_{0}^{(R)} \tilde{q}_{n^{\prime}}^{(R)}+\tilde{d}_{n^{\prime}}^{(R)},
$$

where $\tilde{d}_{n^{\prime}}^{(R)}$ is the time-domain nonlinear distortion noise uncorrelated with $\tilde{q}_{n^{\prime}}^{(R)}$ and $K_{0}^{(R)}$ is a complex-valued constant given by $K_{0}^{(R)}=\mathbb{E}\left[\tilde{z}_{n^{\prime}}^{(R)}\left(\tilde{q}_{n^{\prime}}^{(R)}\right)^{*}\right] / P_{r}$. 


$$
\begin{gathered}
\gamma_{n}^{(S R D)}=\frac{\left|h_{n}^{(R D)} h_{M_{S}}^{(S R)}\right|^{2}\left|K_{0}^{(R)}\right|^{2} P_{r} P_{s}}{\sigma_{v}^{2}\left(\left|h_{M_{S}}^{(S R)}\right|^{2} P_{s}+\left|h_{n}^{(R D)}\right|^{2}\left|K_{0}^{(R)}\right|^{2} P_{r}+\sigma_{v}^{2}\right)+\left|h_{n}^{(R D)}\right|^{2} \sigma_{d^{(R)}}^{2}\left(\left|h_{M_{S}}^{(S R)}\right|^{2} P_{s}+\sigma_{v}^{2}\right)} \\
\gamma_{n}^{(S R D)}=\frac{\gamma_{M_{S}}^{(S R)} \gamma_{n}^{(R D)} \gamma^{(P A)}}{\gamma_{M_{S}}^{(S R)} \gamma_{n}^{(R D)}+\gamma_{M_{S}}^{(S R)} \gamma^{(P A)}+\gamma_{n}^{(R D)} \gamma^{(P A)}+\gamma_{n}^{(R D)}+\gamma^{(P A)}}
\end{gathered}
$$

In the frequency-domain, (4) yields:

$$
\begin{aligned}
z_{n}^{(R)} & =K_{0}^{(R)} q_{n}^{(R)}+d_{n}^{(R)} \\
& =K_{0}^{(R)} g x_{n}^{(S R)}+d_{n}^{(R)}
\end{aligned}
$$

for $1 \leq n \leq N$, where $z_{n}^{(R)}, q_{n}^{(R)}$ and $d_{n}^{(R)}$ are the discrete Fourier transforms (DFTs) of $\tilde{z}_{n^{\prime}}^{(R)}, \tilde{q}_{n^{\prime}}^{(R)}$ and $\tilde{d}_{n^{\prime}}^{(R)}$ $\left(1 \leq n^{\prime} \leq N\right)$, respectively, $d_{n}^{(R)}$ being uncorrelated with $q_{n}^{(R)}$. The signal $d_{n}^{(R)}$ is the frequency-domain nonlinear distortion (NLD). For a rectangular pulse shaping, the NLD is a sum of $N$ uncorrelated complex random variables and it can be modeled as a complex Gaussian random variable with variance [9]: $\sigma_{d^{(R)}}^{2}=\sigma_{\tilde{d}^{(R)}}^{2}=\mathbb{E}\left[\left|z_{n^{\prime}}^{(R)}\right|^{2}\right]-\left|K_{0}^{(R)}\right|^{2} P_{r}$, where $\sigma_{d^{(R)}}^{2}$ and $\sigma_{\tilde{d}(R)}^{2}$ are the variances of $d_{n}^{(R)}$ and $\tilde{d}_{n^{\prime}}^{(R)}$, respectively. From (5), it can be concluded that, in the frequency-domain, the PA can be viewed as a linear system with a fixed gain $K_{0}^{(R)}$ and an additive uncorrelated noise of variance $\sigma_{d^{(R)}}^{2}$. For certain nonlinear functions $F(\cdot)$, there are analytical expressions for $K_{0}^{(R)}$ and $\sigma_{d^{(R)}}^{2}$ [7], [9].

The signal received by the destination in the frequencydomain at the $n^{\text {th }}$ subcarrier during the transmission stage 2 is then given by:

$$
x_{n}^{(R D)}=h_{n}^{(R D)} z_{n}^{(R)}+v_{n}^{(R D)}
$$

where $h_{n}^{(R D)}$ is the CFR at the $n^{t h}$ subcarrier of the selected relay-destination channel and $v_{n}^{(R D)}$ is the AWGN term.

Substituting (2) and (5) into (6), we get:

$$
\begin{aligned}
x_{n}^{(R D)} & =h_{n}^{(R D)} K_{0}^{(R)} g h_{M_{S}}^{(S R)} \sqrt{P_{s}} s_{n} \\
& +h_{n}^{(R D)} K_{0}^{(R)} g v_{n}^{(S R)}+h_{n}^{(R D)} d_{n}^{(R)}+v_{n}^{(R D)},
\end{aligned}
$$

or, equivalently:

$$
x_{n}^{(R D)}=h_{n}^{(S R D)} s_{n}+v_{n}^{(S R D)},
$$

where $h_{n}^{(S R D)}=h_{n}^{(R D)} K_{0}^{(R)} g h_{M_{S}}^{(S R)} \sqrt{P_{s}}$ and

$$
v_{n}^{(S R D)}=h_{n}^{(R D)} K_{0}^{(R)} g v_{n}^{(S R)}+h_{n}^{(R D)} d_{n}^{(R)}+v_{n}^{(R D)} .
$$

From (2), (5) and (6), note that the source-relay-destination link can be viewed as a three-hop linear relaying channel. In other words, nonlinear PA acts as another linear relaying channel, placed between the relay and the destination.

\section{OUtAge AnALYsis}

In this Section, an expression for the outage probability of the considered AF OFDM system is derived. Herein, we denote by $f_{X}(\cdot)$ and $F_{X}(\cdot)$ the probability density function (PDF) and cumulative distribution function (CDF) of the random variable $X$, respectively.

\section{A. Instantaneous Signal-to-Noise Ratio}

The instantaneous SNR after the MRC is given by $\gamma_{n}=$ $\gamma_{n}^{(S D)}+\gamma_{n}^{(S R D)}$, with $\gamma_{n}^{(S D)}=\left|h_{n}^{(S D)}\right|^{2} P_{s} / \sigma_{v}^{2}$ and $\gamma_{n}^{(S R D)}=$ $\left|h_{n}^{(S R D)}\right|^{2} / \sigma_{v_{n}^{\prime}}^{2}$, where $\sigma_{v_{n}^{\prime}}^{2}$ is the variance of $v_{n}^{(S R D)}$ given by:

$$
\sigma_{v_{n}^{\prime}}^{2}=\sigma_{v}^{2}\left(1+\left|h_{n}^{(R D)}\right|^{2}\left|K_{0}^{(R)}\right|^{2} g^{2}\right)+\left|h_{n}^{(R D)}\right|^{2} \sigma_{d^{(R)}}^{2} .
$$

In [6], this type of MRC receiver is called NLD-aware MRC, as the receiver must be aware of the NLD to carry out the MRC. From (10), it follows that:

$$
\gamma_{n}^{(S R D)}=\frac{\left|h_{n}^{(R D)} h_{M_{S}}^{(S R)}\right|^{2} g_{n}^{2}\left|K_{0}^{(R)}\right|^{2} P_{s}}{\sigma_{v}^{2}\left(1+\left|h_{n}^{(R D)}\right|^{2}\left|K_{0}^{(R)}\right|^{2} g_{n}^{2}\right)+\left|h_{n}^{(R D)}\right|^{2} \sigma_{d^{(R)}}^{2}} .
$$

Then, using the variable gain expression, (12) is obtained, given at the top of this page.

By its turn, defining the following wireless links instantaneous SNRs: $\gamma_{M_{S}}^{(S R)}=\left|h_{M_{S}}^{(S R)}\right|^{2} P_{s} / \sigma_{v}^{2}$ and $\gamma_{n}^{(R D)}=$ $\left|h_{n}^{(R D)}\right|^{2}\left|K_{0}^{(R)}\right|^{2} P_{r} / \sigma_{v}^{2}$, and the instantaneous SNR at the output of the PA as: $\gamma^{(P A)}=\left|K_{0}^{(R)}\right|^{2} P_{r} / \sigma_{d^{(R)}}^{2}$, (12) can be rewritten as (13), where the latter corresponds to the instantaneous SNR of a three-hop cooperative AF system. Indeed, by defining:

$$
\gamma_{n}^{(P A, R D)}=\frac{\gamma^{(P A)} \gamma_{n}^{(R D)}}{\gamma^{(P A)}+\gamma_{n}^{(R D)}},
$$

(13) can be expressed as:

$$
\gamma_{n}^{(S R D)}=\frac{\gamma_{M_{S}}^{(S R)} \gamma_{n}^{(P A, R D)}}{\gamma_{M_{S}}^{(S R)}+\gamma_{n}^{(P A, R D)}+1} .
$$

Note that (15) corresponds to the instantaneous SNR of a twohop cooperative AF system with individual link SNRs given by $\gamma_{M_{S}}^{(S R)}$ and $\gamma_{n}^{(P A, R D)}$, and a variable relay gain given by $g$, while (14) corresponds to the instantaneous SNR of a two-hop system with individual link SNRs given by $\gamma^{(P A)}$ and $\gamma_{n}^{(R D)}$, and a relay gain given by $1 /\left|K_{0}^{(R)}\right|[10]$.

The SNR $\gamma_{n}$ can be approximated by the following upper bound [10], [11]:

$$
\gamma_{n}=\gamma_{n}^{(S D)}+\min \left(\gamma_{M_{S}}^{(S R)}, \gamma^{(P A)}, \gamma_{n}^{(R D)}\right) .
$$

It is noteworthy that the approximation above is adopted in many previous works that investigate multihop relaying systems [10], [11]. As it will be shown in the simulation results section, such an approximation also applies to our work, being reasonably accurate for low and high SNRs. 


\section{B. Outage Probability}

Defining the outage probability of the $n^{\text {th }}$ subcarrier of the considered OFDM system as: $P_{n}^{(o u t)}\left(\gamma_{t h}\right)=\operatorname{Prob}\left(\gamma_{n}<\gamma_{t h}\right)$, for $\gamma_{n}, \gamma_{t h} \geq 0$, where $\gamma_{n}$ is the instantaneous SNR given by (15) and $\gamma_{t h}$ is a threshold value, we can write:

$$
\begin{aligned}
P_{n}^{(\text {out })}\left(\gamma_{t h}\right) & =\operatorname{Prob}\left(\gamma_{n}^{(S D)}+\gamma_{n}^{(\min )}<\gamma_{t h}\right) \\
& =\int_{0}^{+\infty} f_{\gamma_{n}^{(S D)}}(x)\left[\int_{0}^{\gamma_{t h}-x} f_{\gamma_{n}^{(\min )}}(y) d y\right] d x \\
& =\int_{0}^{+\infty} f_{\gamma_{n}^{(S D)}}(x) F_{\gamma_{n}^{(\min )}}\left(\gamma_{t h}-x\right) d x
\end{aligned}
$$

where $\gamma_{n}^{(\min )}=\min \left(\gamma_{M_{S}}^{(S R)}, \gamma^{(P A)}, \gamma_{n}^{(R D)}\right)$ and:

$$
\begin{aligned}
& F_{\gamma_{n}^{(m i n)}}\left(\gamma_{t h}\right)=1- \\
& {\left[1-F_{\gamma_{M_{S}}^{(S R)}}\left(\gamma_{t h}\right)\right]\left[1-F_{\gamma^{(P A)}}\left(\gamma_{t h}\right)\right]\left[1-F_{\gamma_{n}^{(R D)}}\left(\gamma_{t h}\right)\right] .}
\end{aligned}
$$

Besides, $\gamma_{M_{S}}^{(S R)}=\max \left(\gamma_{1}^{(S R)}, \gamma_{2}^{(S R)}, \ldots, \gamma_{M}^{(S R)}\right)$, where $\gamma_{m}^{(S R)}=P_{s}\left|h_{m}^{(S R)}\right|^{2} / \sigma_{v}^{2}$, for $1 \leq m \leq M$, which leads to:

$$
F_{\gamma_{M_{S}}^{(S R)}}\left(\gamma_{t h}\right)=F_{\gamma_{1}^{(S R)}}\left(\gamma_{t h}\right) \ldots F_{\gamma_{M}^{(S R)}}\left(\gamma_{t h}\right)
$$

Substituting (19) into (18), we get:

$$
\begin{array}{r}
F_{\gamma_{n}^{(m i n)}}\left(\gamma_{t h}\right)=1-\left[1-\prod_{r=1}^{M} F_{\gamma_{r}^{(S R)}}\left(\gamma_{t h}\right)\right] \\
{\left[1-F_{\gamma^{(P A)}}\left(\gamma_{t h}\right)\right]\left[1-F_{\gamma_{n}^{(R D)}}\left(\gamma_{t h}\right)\right] .}
\end{array}
$$

Since Rayleigh fading is considered, the instantaneous SNRs of the wireless links are exponentially distributed, that is, $F_{\gamma_{m}^{(S R)}}\left(\gamma_{t h}\right)=\left[1-\exp \left(-\gamma_{t h} / \bar{\gamma}^{(S R)}\right)\right] u\left(\gamma_{t h}\right)$ and $F_{\gamma_{n}^{(R D)}}\left(\gamma_{t h}\right)=\left[1-\exp \left(-\gamma_{t h} / \bar{\gamma}^{(R D)}\right)\right] u\left(\gamma_{t h}\right)$, where $u(\cdot)$ is the unit step function, and $\bar{\gamma}^{(R D)}$ and $\bar{\gamma}^{(S R)}$ denote respectively the standard deviations of $\gamma_{n}^{(R D)}$ and $\gamma_{m}^{(S R)}$, for $1 \leq m \leq M$. Moreover, let us consider that the PA SNR is fixed, that is, $F_{\gamma^{(P A)}}\left(\gamma_{t h}\right)=u\left(\gamma_{t h}-\bar{\gamma}^{(P A)}\right)$, where $\bar{\gamma}^{(P A)}=$ $\left|K_{0}^{(R)}\right|{ }^{2} P_{r} / \sigma_{d^{(R)}}^{2}$. Thus, (20) can be re-expressed as:

$$
\begin{array}{r}
F_{\gamma_{n}^{(m i n)}}\left(\gamma_{t h}\right)=1-\left[1-\left(1-e^{\frac{-\gamma_{t h}}{\bar{\gamma}^{(S R)}}}\right)^{M} u\left(\gamma_{t h}\right)\right] \\
{\left[u\left(-\gamma_{t h}\right)+e^{\frac{-\gamma_{t h}}{\bar{\gamma}^{(R D)}}} u\left(\gamma_{t h}\right)\right] u\left(\bar{\gamma}^{(P A)}-\gamma_{t h}\right)}
\end{array}
$$

Making use of the binomial expansion, it follows that:

$$
\begin{array}{r}
F_{\gamma_{n}^{(\text {min })}}\left(\gamma_{t h}\right)=1-\left[u\left(-\gamma_{t h}\right)-\sum_{m=1}^{M}\left(\begin{array}{c}
M \\
m
\end{array}\right)(-1)^{m}\right. \\
\left.e^{\frac{-\gamma_{t h} m}{\bar{\gamma}^{(S R)}}} u\left(\gamma_{t h}\right)\right]\left[u\left(-\gamma_{t h}\right)+e^{\frac{-\gamma_{t h}}{\bar{\gamma}^{(R D)}}} u\left(\gamma_{t h}\right)\right] \\
u\left(\bar{\gamma}^{(P A)}-\gamma_{t h}\right) .
\end{array}
$$

Defining $\bar{\gamma}_{m}=\left(\frac{m}{\bar{\gamma}^{(S R)}}+\frac{1}{\bar{\gamma}^{(R D)}}\right)^{-1}$ and $\beta_{M, m}=\left(\begin{array}{l}M \\ m\end{array}\right)(-1)^{m}$, (22) can be rewritten as:

$$
\begin{aligned}
& F_{\gamma_{n}^{(m i n)}}\left(\gamma_{t h}\right)=1-\left[u\left(-\gamma_{t h}\right)\right. \\
& \left.-u\left(\gamma_{t h}\right) \sum_{m=1}^{M} \beta_{M, m} e^{-\gamma_{t h} / \bar{\gamma}_{m}}\right] u\left(\bar{\gamma}^{(P A)}-\gamma_{t h}\right),
\end{aligned}
$$

or, equivalently:

$$
\begin{array}{r}
F_{\gamma_{n}^{(m i n)}}\left(\gamma_{t h}\right)=u\left(\gamma_{t h}\right)+\left(\sum_{m=1}^{M} \beta_{M, m} e^{-\gamma_{t h} / \bar{\gamma}_{m}}\right) u\left(\gamma_{t h}\right) \\
u\left(\bar{\gamma}^{(P A)}-\gamma_{t h}\right) .
\end{array}
$$

Then, plugging (24) into (17), we get:

$$
\begin{array}{r}
P^{(\text {out })}\left(\gamma_{t h}\right)=\int_{0}^{+\infty}\left[u\left(\gamma_{t h}-x\right)+u\left(\gamma_{t h}-x\right)\right. \\
\left.u\left(\bar{\gamma}^{(P A)}-\gamma_{t h}+x\right)\left(\sum_{m=1}^{M} \beta_{M, m} e^{-\left(\gamma_{t h}-x\right) / \bar{\gamma}_{m}}\right)\right] \\
f_{\gamma_{n}^{(S D)}}(x) d x,
\end{array}
$$

for $\gamma_{t h} \geq 0$, which leads to:

$$
\begin{array}{r}
P^{\text {(out })}\left(\gamma_{t h}\right)=F_{\gamma_{n}^{(S D)}}\left(\gamma_{t h}\right)+\sum_{m=1}^{M} \beta_{M, m} e^{-\gamma_{t h} / \bar{\gamma}_{m}} \\
\int_{\max \left(\gamma_{t h}-\bar{\gamma}^{(P A)}, 0\right)}^{\gamma_{t h}} f_{\gamma_{n}^{(S D)}(x) e^{x / \bar{\gamma}_{m}} d x .}
\end{array}
$$

As the outage probability in (25) and (26) does not depend on the subcarrier, the index $n$ was omitted from $P_{n}^{(o u t)}\left(\gamma_{t h}\right)$.

Since $\gamma_{n}^{(S D)}$ is exponentially distributed with standard deviation $\bar{\gamma}^{(S D)}$, it follows that:

$$
\begin{gathered}
P^{(\text {out })}\left(\gamma_{t h}\right)=F_{\gamma_{n}^{(S D)}}\left(\gamma_{t h}\right)+\frac{1}{\bar{\gamma}^{(S D)}} \sum_{m=1}^{M} \beta_{M, m} e^{-\gamma_{t h} / \bar{\gamma}_{m}} \\
\int_{\max \left(\gamma_{t h}-\bar{\gamma}^{(P A)}, 0\right)}^{\gamma_{t h}} e^{x / \bar{\gamma}_{m}^{(S R D)}} d x,
\end{gathered}
$$

where $\bar{\gamma}_{m}^{(S R D)}=\left(1 / \bar{\gamma}_{m}-1 / \bar{\gamma}^{(S D)}\right)^{-1}$, leading to:

$$
\begin{array}{r}
P^{(\text {out })}\left(\gamma_{t h}\right)=F_{\gamma_{n}^{(S D)}}\left(\gamma_{t h}\right)+\sum_{m=1}^{M} \frac{\bar{\gamma}_{m}^{(S R D)}}{\bar{\gamma}^{(S D)}} \beta_{M, m} e^{-\gamma_{t h} / \bar{\gamma}_{m}} \\
{\left[e^{\frac{\gamma_{t h}}{\bar{\gamma}_{m}^{(S R D)}}}-e^{\frac{\max \left(\gamma_{t h}-\bar{\gamma}^{(P A)}, 0\right)}{\bar{\gamma}_{m}^{(S R D)}}}\right] .}
\end{array}
$$

By its turn, (28) can be rewritten as:

$$
\begin{aligned}
P^{(\text {out })}\left(\gamma_{t h}\right)=1-e^{-\frac{\gamma_{t h}}{\bar{\gamma}^{(S D)}}}+\sum_{m=1}^{M} \frac{\bar{\gamma}_{m}^{(S R D)}}{\bar{\gamma}^{(S D)}} \beta_{M, m} e^{-\gamma_{t h} / \bar{\gamma}_{m}} \\
{\left[1-e^{-\frac{\min \left(\gamma_{t h}, \bar{\gamma}^{(P A)}\right)}{\bar{\gamma}_{m}^{(S R D)}}}\right] e^{\frac{\gamma_{t h}}{\bar{\gamma}_{m}^{(S R D)}}}, }
\end{aligned}
$$

which leads to the following outage probability expression:

$$
\begin{aligned}
P^{(\text {out })}\left(\gamma_{t h}\right)=1- & {\left[1-\sum_{m=1}^{M} \frac{\bar{\gamma}_{m}^{(S R D)}}{\bar{\gamma}^{(S D)}} \beta_{M, m}\right.} \\
& \left.\left(1-e^{-\frac{\min \left(\gamma_{t h}, \bar{\gamma}^{(P A)}\right)}{\bar{\gamma}_{m}^{(S R D)}}}\right)\right] e^{-\frac{\gamma_{t h}}{\bar{\gamma}^{(S D)}}} .
\end{aligned}
$$

Note that the outage probability in (30) depends on the nonlinear PA parameters only for $\gamma_{t h}>\bar{\gamma}^{(P A)}$. Besides, if $\gamma_{t h}>\bar{\gamma}^{(P A)}, P_{n}^{(\text {out })}\left(\gamma_{t h}\right)$ decreases slowly with an increase of $\gamma_{t h}$ than if $\bar{\gamma}^{(P A)}>\gamma_{t h}$. This means that the PA nonlinearity 


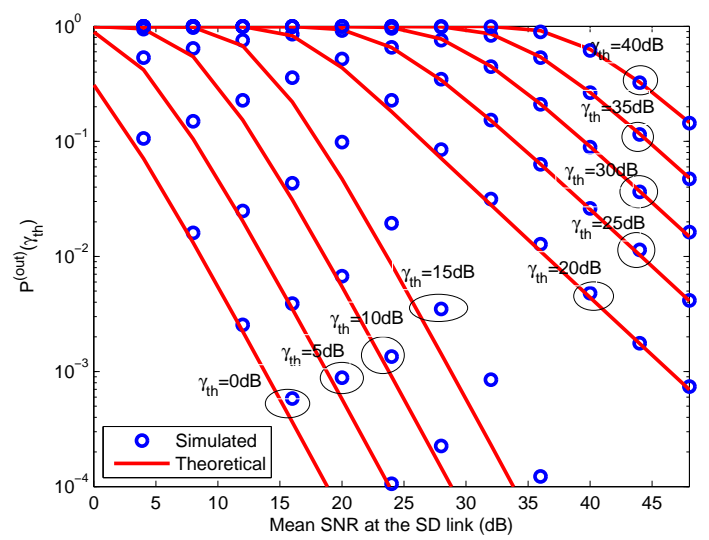

Fig. 2. $P_{n}^{(\text {out })}\left(\gamma_{t h}\right)$ versus the mean SNR for various values of $\gamma_{t h}$.

increases the outage probability for high SNR thresholds $\gamma_{t h}$, but it does not change the outage probability for small $\gamma_{t h}$. Moreover, when there is no direct path between source and destination, the outage probability is given by: $P_{n}^{(o u t)}\left(\gamma_{t h}\right)=$ $F_{\gamma_{n}^{(m i n)}}\left(\gamma_{t h}\right)$ in (24).

\section{Simulation Results}

In this section, the validity of the outage probability expression developed in Section III is evaluated by means of simulations. An AF cooperative OFDM system with $R$ available relays and Rayleigh fading channels has been considered for the simulations, with the relay PA being modeled by a soft clipper (soft limiter). Closed-form expressions for $K_{0}^{(R)}$ and $\sigma_{d^{(R)}}^{2}$ are given in [7], [9] for the case of a soft clipping PA. Moreover, $P_{r}=P_{s}=0.5$, which leads to $\bar{\gamma}^{(P A)}=17.5 \mathrm{~dB}$. The results were obtained with $N=256$ subcarriers and QPSK (quadrature PSK) transmitted signals, via Monte Carlo simulations. It is assumed that the destination knows perfectly all the channels and that the relay knows perfectly the sourcerelay channel.

Fig. 2 shows the outage probability versus the mean SNR at the source-destination link, the outage probability being obtained by means of simulations and using (30), for various values of $\gamma_{t h}$ and $M=4$. It can be noted that the theoretical and simulated curves are very close. Moreover, by the slopes of the curves, it can be concluded that the system diversity is higher when $\gamma_{t h}<\bar{\gamma}^{(P A)}=17.5 \mathrm{~dB}$. This is due to the fact the NLD is more significant for high channel SNRs than for lower SNRs.

Fig. 3 shows the theoretical outage probability versus the mean SNR at the source-destination link, for various values of $\gamma_{t h}$, with $M=4$ and $M=1$. It is also shown the values of $P_{n}^{(\text {out })}\left(\gamma_{t h}\right)$ obtained with a linear PA and $M=4$. It can be viewed that, when $M=4$ relays are available, the relay selection provides a SNR gain of approximately $1.5 \mathrm{~dB}$ with respect to the case $M=1$, for $\gamma_{t h}<\bar{\gamma}^{(P A)}$. However, for $\gamma_{t h}>\bar{\gamma}^{(P A)}$, the relay selection does not provide a performance gain, due to the fact that, in this situation, the NLD is the bottleneck of the system performance. Moreover, as expected, the nonlinear PA affects the outage probability only for $\gamma_{t h}>\bar{\gamma}^{(P A)}$.

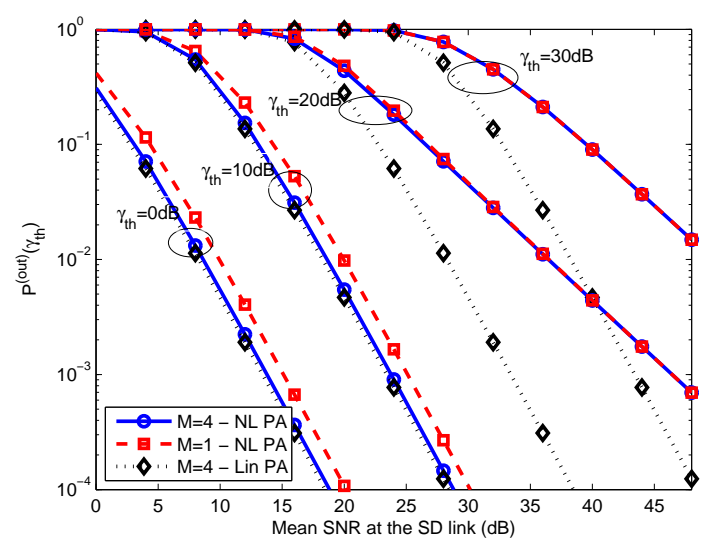

Fig. 3. $P_{n}^{(\text {out })}\left(\gamma_{t h}\right)$ versus the mean SNR for linear and nonlinear PAs.

\section{CONCLUSION}

A theoretical outage analysis of an AF cooperative OFDM system with a nonlinear PA and relay selection has been developed in this paper. An outage probability expression has been derived by approximating the instantaneous SNR of the MRC output by an upper bound. Simulation results have shown that the developed outage probability expression has good accuracy and that relay selection provides performance gains only for small SNR thresholds. Moreover, simulations have shown that the nonlinear PA decreases the system diversity only for high SNR thresholds. In future works, the analysis of this paper should be extended to frequency-selective channels, as well as to other relay selection methods.

\section{REFERÊNCIAS}

[1] J. N. Laneman, D. N. C. Tse, and G. W. Wornell, "Cooperative diversity in wireless networks: efficient protocols and outage behavior," IEEE Trans. Inf. Theory, vol. 50, no. 12, pp. 3062-3080, Dec. 2004.

[2] K. J. Ray Liu, A. K. Sadek, W. Su, and A. Kwasinski, Cooperative communications and Networking, Cambridge University Press, 2009.

[3] A. Bletsas, A. Khisti, D. P. Reed, and A. Lippman, "A simple cooperative diversity method based on network path selection," IEEE J. Select. Areas Commun., vol. 24, no. 3, pp. 659-672, Mar. 2006.

[4] I. Krikidis, J. Thompson, S. McLaughlin, and N. Goertz, "Amplify-andforward with partial relay selection," IEEE Commun. Lett., vol. 12, no. 4, pp. 235-237, Apr. 2008.

[5] T. Hwang, C. Yang, G. Wu, S. Li, and G. Y. Li, "OFDM and its wireless applications: a survey," IEEE Trans. Veh. Technol., vol. 58, no. 4, pp. 1673-1694, May 2009.

[6] V. del Razo, T. Riihonen, F. H. Gregorio, S. Werner, and R. Wichman, "Nonlinear amplifier distortion in cooperative amplify-and-forward OFDM systems," in IEEE Wireless Commun. Net. Conf. (WCNC), Budapest, Hungary, Apr. 2009.

[7] T. Riihonen, S. Werner, F. Gregorio, R. Wichman, and J. Hamalainen, "BEP analysis of OFDM relay links with nonlinear power amplifiers," in IEEE Wireless Commun. Net. Conf. (WCNC), Sydney, Australia, Apr. 2010, pp. 1-6.

[8] C. Zhang, P. Ren, J. Peng, G. Wei, Q. Du, and Y. Wang, "Optimal relay power allocation for amplify-and-forward relay networks with non-linear power amplifiers," CoRR, vol. abs/1105.0116, Apr. 2011.

[9] D. Dardari, V. Tralli, and A. Vaccari, "A theoretical characterization of nonlinear distortion effects in OFDM systems," IEEE Trans. Commun., vol. 48 , no. 10 , pp. $1755-1764$, Oct. 2000.

[10] M. O. Hasna and M.-S. Alouini, "Outage probability of multihop transmission over nakagami fading channels," IEEE Commun. Lett., vol. 5, no. 7, pp. 1089-7798, May 2003.

[11] S. Ikki and M. A. Ahemd, "Performance analysis of cooperative diversity wireless networks over nakagami-m fading channel," IEEE Commun. Lett., vol. 11, no. 4, pp. 334-336, Apr. 2007. 